\title{
An EOQ model for deteriorating items when quantity received uncertain under permissible delay in payment with planned shortages and equivalent holding cost and shortages cost
}

\author{
Raina Pandya $^{1}$ and Chirag J Trivedi ${ }^{2}$ \\ ${ }^{1}$ Research scholar, statistic Department, Gujarat University, \\ Ahmedabad, India \\ ${ }^{2}$ Head \& Associate professor, statistic Department, R.J.Tibrewal Commerce College, \\ Ahmedabad, India.
}

\begin{abstract}
In this paper, derive new deterministic inventory model with equivalent holding and shortages cost, under the planned expected shortages of quantity and delay in payment for deteriorating items when quantity received is uncertain. The sensitivity analysis to study interdependence of parameters on the decision variables and objective function for derived model is explain by hypothetical numerical illustration
\end{abstract}

Key words: $E O Q$, uncertain quantity, delay in payments, deteriorating items, equivalent holding and shortages cost, planned shortages.

\section{Introduction:}

In the classical inventory EOQ model, quantity received uncertain means there is no any equality between quantity received and the quantity requisitioned and there is no damage or deterioration of units while in inventory and also that the supplier must be paid for the goods as soon as they are received by the inventory system. There are deferent situations and strategies in inventory system. An EOQ model dealing mainly with holding cost and shortages cost. This model depends on planned shortages and dealing with the equivalent holding and shortages cost. Also it is normal practice in business and market that the wholesaler tolerates assured fixed credit policy period for manage the balance sheet of profit and no any interest charges are payable if the balance sheet is settled within the decided period.

Silver (1976) has settled an EOQ model when the quantity received is uncertain and is a random variable with stated mean and variance. Kalro and Gohil (1982) have expand the above model by allowing shortages. Noori and Keller (1986) developed a stochastic model when quantity received is uncertain. Ghare and Schrader (1963) developed an EOQ model for exponentially decaying inventories. The model has been generalized by Covert and Philip (1973) and the by Philip (1974) by using weibull distribution to define time to deterioration of an inventory. Goyal (1985) has established an EOQ model when supplier allows fixed credit period for settling the accounts. Mandal and Phaujdar (1989) generalized the work of Goyal (1985) by taking into consideration a variety of accurate situations. Kingsman (1997) planned the relationship between the inventory cost and payment rules. Chang (2002) discussed an inventory model with time dependent deterioration rate considering the effect of permissible delay in payment. Rao and Rao (2015) analyzed an inventory model for deterioration item with permissible delay in payment. Ghosh and Chaudhuri (2004) study an order level inventory model with shortages. Eoq for planned shortages it seen in very few books (gupta 2008, Narsimha 2010, Sharma 2010, and Vora 2011). Some authors use expressions of back ordering while many authors go with the planned shortages to derive model. Here prefer the period of 
planned shortages for the EOQ model when stock extents maximum planned shortages. Equivalent holding cost really makes things easier the traditional inventory model (Kharde. Vikhe, Patil, 2011a, 2011b) . We derive the model equivalent holding cost when quantity received uncertain and it's just parallel using eoq formulas.

In this paper, we analyze an inventory EOQ model for planned shortages when the quantity received uncertain and the delay in payment is permissible. During the time account is not settled, it is expected that the cost of unit sold is deposited in an interest manner accounts and the profit margin is used to meet the further operational expenses of the inventory system. However the cause happen shortages of a stock planned that the quantity order and received no equality. The supplier must have to pay for the goods purchase as early as possible. Also routine business that the supplier allow certain fixed time for settling account and there is no interest charges if account settle within the fixed period of time.

\section{Notations:}

$\mathrm{Q}=$ the order quantity

$\mathrm{Q}^{*}=$ Economic order quantity

$\mathrm{C}_{3}=$ Replenishment cost per order

Ic $=$ The interest charges per rupee investment in stock per time unit.

$\mathrm{Ie}=$ the interest that can be earned per rupee per time unit

$\mathrm{T}^{*}=$ permissible delay in payment in time unit

$\mathrm{T}(\mathrm{Q})=$ Total annual inventory

$\mathrm{d}=$ uniform demand

$\mathrm{R}=$ Inventory fix rate

LT = Lead Time

$\mathrm{A}=$ Re-order level

$F_{b}=$ Factor for equivalent holding cost for back and shortages cost

$\mathrm{EC}_{1} \mathrm{C}_{2}=$ Equivalent Holding and shortages cost when Back-order are permitted under permissible delay in payment.

$\mathrm{M}=$ Maximum Inventory level units

$\mathrm{S}=$ Maximum shortages level or maximum backorder quantity unit
$\mathrm{D}=$ Annual demand units.

$\mathrm{N}=$ Number of order per year.

TEC $(\mathrm{Q})=$ Total expected cost per time unit

Q-S = Shortages before quantity added.

\section{Assumptions}

1. The demand Rate of D units per time unit is known and constant.

2. Lead time is fixed

3. Holding cost $\left(\mathrm{C}_{1}\right)$ per unit per unit time is constant and does not change for differ order quantity

4. Shortages $\left(\mathrm{C}_{2}\right)$ per unit per time is known and constant

5. Time horizon is infinite

6. Ordering cost $\left(\mathrm{C}_{3}\right)$ per order is known and constant.

7. The unit cost $\mathrm{C}$ does not depend upon the quantity ordered or received.

8. Purchase price of the item is constant and does not change with delay in payment. Number of discount is applicable.

9. The replenishment is infinite for order quantity is done when shortages level reaches planned shortages level in one lot.

10. The supplier gives a listed credit period of $\mathrm{T}^{*}$ time unit.

11. Stock out are acceptable and shortages or back ordering cost per unit is known and constant.

12. The quantity requisitioned does not necessary match with the quantity received. It $\mathrm{Q}$ does not he quantity requisitioned and $\mathrm{Y}$ is the quantity received then $\mathrm{Y}$ is a random Variable with $\mathrm{E}(\mathrm{Y})=\mathrm{bQ}$ and $\mathrm{V}(\mathrm{Y})=\sigma_{0}{ }^{2}+\left(\sigma_{1}{ }^{2}+\mathrm{b}^{2}\right)$ $\mathrm{Q}^{2}$ when $\mathrm{b}>0$ is the bias factor and $\sigma_{0}^{2}$, $\sigma_{1}{ }^{2}>0$ are constant

13. At time $\mathrm{T}$ of a cycle there fraction $\theta$ on hand inventory deteriorates per unit of time.

14. There is no repair or replenishment of the deteriorated inventory during the period under consideration.

\section{Mathematical model: An EOQ model for deteriorating items under permissible delay in payment}

Let $\mathrm{Q}(\mathrm{t})$ denotes the on hand inventory during planned shortages at time $\mathrm{t}$ of a cycle and $\mathrm{Y}$ is expected uncertain quantity. Where $\mathrm{T}=\mathrm{T}(\mathrm{Y})=\frac{Y}{R}$ is cycle time. Then the differential equation that defines the instantons states of method is 


$$
\begin{aligned}
& \frac{d(Q(t))}{d t}=-R \quad o \leq t \leq T \\
& \therefore \quad \mathrm{Q}(\mathrm{t})=\mathrm{R}(\mathrm{T}-\mathrm{t})
\end{aligned}
$$

Here, $\left[R T=Y \therefore T=\frac{Y}{R}\right]$

$$
\mathrm{Q}(\mathrm{t})=\mathrm{Y}-\mathrm{RT}
$$

\section{Holding cost: $\mathrm{C}_{1}$ for deteriorating items}

Inventory demand rate $: \mathrm{d}$ when time $: \mathrm{t}$

Here, $Q=$ order quantity;

So, Maximum inventory: $\mathrm{M}=\mathrm{Q}-\mathrm{S}=\mathrm{d} . \mathrm{t}$ Average inventory during $\mathrm{t}_{1}=$

$$
\frac{d . t}{2}=\frac{Q-S}{2}
$$$$
\mathrm{M}=\mathrm{d} . \mathrm{t} ; \mathrm{Q}=\mathrm{d} . \mathrm{T}
$$

$$
\begin{aligned}
\frac{M}{Q}=\frac{d t}{d T} & =\frac{t}{T} ; N=\frac{1}{T} ; N T=1_{(1)} \\
\mathrm{MT} & =\mathrm{Q}(\mathrm{t})
\end{aligned}
$$

Inventory Quantity is

$$
\begin{aligned}
\mathrm{Q}(\mathrm{t}) & =\int_{0}^{T} \mathrm{R}(\mathrm{T}-\mathrm{t}) \mathrm{dt} \text { OR } \\
\mathrm{Q}(\mathrm{t}) & =\int_{0}^{T} \mathrm{R}(\mathrm{T}-\mathrm{t}) \mathrm{dt} \\
& =\mathrm{Y}^{2}-\mathrm{Rt}
\end{aligned}
$$

Put the values of $\mathrm{Q}(\mathrm{t})$

$$
\begin{aligned}
\mathrm{MT} & =\mathrm{Y}^{2}-\mathrm{Rt} \\
\mathrm{Rt} & =\mathrm{Y}^{2}-\mathrm{MT} \\
& =\mathrm{Y}^{2}-(\mathrm{Q}-\mathrm{S}) \mathrm{T} \\
\mathrm{t} & =\frac{Y^{2}-(Q-S) T}{R}
\end{aligned}
$$

\section{Holding cost during time $t$}

$\mathrm{C}_{1}(\mathrm{Q})=($ Avg. inventory) (Inventory Cost during $\mathrm{t}$ )
$\mathrm{C}_{1}(\mathrm{Q})=\frac{(Q-S)}{2}\left(\mathrm{C}_{1}+\mathrm{C} \theta\right) \mathrm{t}$

\section{Annual holding cost:}

$$
\begin{aligned}
\mathrm{C}_{1}(\mathrm{Q}) & =\left(\frac{\text { Holding cost per Cycle }}{\text { Cycle per year }}\right) \\
& =\left[\frac{Q-S}{2}\right] \quad\left[\left(\mathrm{C}_{1+} \mathrm{C} \theta\right) \mathrm{t}\right] \mathrm{N}
\end{aligned}
$$

$\therefore\left[\frac{Q-S}{2}\right]\left(C_{1}+C \theta\right)\left[\frac{Y^{2}-(Q-S)}{R} T\right] N$

$$
\begin{aligned}
& \mathrm{C}_{1} \\
& {\left[\frac{\left(C_{1}+C \theta\right)\left[Y^{2}-(Q-S)\right]}{2 R}\right]}
\end{aligned}
$$

Shortages cost $\left(\mathrm{C}_{2}\right)$ : for deteriorating items

When time $=\mathrm{t}$ and shortages rate: $\mathrm{d}$ Maximum shortages $=\mathrm{S}=\mathrm{d} \mathrm{t}$

Average shortages during $=\frac{d t}{2}=\frac{S}{2}$ when time: $\mathrm{t}$

$\mathrm{S}=\mathrm{d} \mathrm{t} \quad \& \mathrm{Q}=\mathrm{d} . \mathrm{T}$.

$\frac{S}{Q}=\frac{d t}{d T}=\frac{t}{T} \quad S=\frac{Q(t)}{T}$

$\mathrm{ST}=\mathrm{Q}(\mathrm{t})=\mathrm{Y}-\mathrm{RT}$

$$
t=\frac{Y^{2}-S T}{R}
$$

$\mathrm{C}_{2}(\mathrm{Q})=$ (average shortages)

$\left(\frac{\text { shortages } \cos t}{\text { unit timet }}\right)$

$$
=\frac{S}{2} \cdot\left(C_{2}+C \theta\right) t
$$

\section{Annual shortages cost:}




$$
\begin{aligned}
& \left(\frac{\text { shortages } \cos t}{\text { cycle }}\right)(\text { cycleperyear }) \\
& C_{2}(Q)=\frac{S}{2}\left[\left(C_{2}+C \theta\right) \cdot t\right] \cdot N \\
& =\left(\frac{S}{2}\right)\left(C_{2}+C \theta\right)\left(\frac{Y^{2}-S T}{R}\right) \cdot N \\
& C_{2}(Q)=\frac{\left(C_{2}+C \theta\right) \cdot\left(Y^{2}-S\right)}{2 R}
\end{aligned}
$$

Ordering cost $=\mathrm{C}_{3} \quad(\mathrm{Q})=$ $\frac{\text { annual demand }}{\text { unit order Quantity }}=\frac{D}{Q}$

Annual ordering cost $=\mathrm{C}_{3}(\mathrm{Q})=$ $\left(\frac{\text { order }}{\text { year }}\right)($ ordering $\cos t)$

$$
=\frac{D}{Q} \cdot \mathrm{C}_{3}
$$

\section{We analyze one cycle two cases may arise:}

The net (annual) cost of the structure is given by

$$
\begin{aligned}
& \begin{array}{l}
\mathrm{K}_{1}(\mathrm{Y} / \mathrm{Q})= \\
\frac{\left(C_{1}+C \theta\right)\left[Y^{2}-(Q-S)\right]}{2 R}+\frac{C I c\left[\theta\left(Y-R T^{*}\right)^{2}\right]}{2 R}+\frac{\left(C_{2}+C \theta\right)\left(y^{2}-S\right)}{2 R}+\frac{D}{Q} C_{3}-\frac{C I e R T^{* 2}}{2}-\text { (2) } \\
=\frac{\left[C_{1}+C \theta+C I c\right]\left[Y^{2}-(Q-S)\right]}{2 R}-\left[\theta\left(C I c T^{*} Y\right)\right]+\frac{\left(C_{2}+C \theta\right)\left(Y^{2}-S\right)}{2 R}+\frac{D}{Q} C_{3}+\frac{C[I c-I e] R T^{* 2}}{2}- \\
=\frac{y^{2}}{2 R}\left[\left(C_{1}+C \theta+C I c\right)-(Q-S)+. C_{2}(1-S)\right]-\theta\left(C I c T^{*} Y\right)+\frac{D}{Q} C_{3}+C[I c-I e] R *^{2}-(4)
\end{array}
\end{aligned}
$$

Therefore we proceeds equivalent Holding and shortages cost

$$
\begin{aligned}
\mathrm{E} \mathrm{C}_{1} \cdot \mathrm{C}_{2} & =\mathrm{C} 1(\mathrm{Q})+\mathrm{C}_{2}(\mathrm{Q}) \\
& =\frac{\left(C_{1}+C \theta\right)\left(Y^{2}-(Q-S)\right)}{2 R}+\frac{\left(C_{2}+C \theta\right)\left(Y^{2}-S\right)}{2 R}
\end{aligned}
$$




$$
=Y^{2}\left[\frac{\left(C_{1}+C \theta\right)(1-(Q-S))}{2 R}+\frac{\left(C_{2}+C \theta\right)(1-S)}{2 R}\right]
$$

Taking expatiation an equations,

$$
E\left(E C_{1} C_{2}\right)=\left[\sigma_{0}^{2}+\left(\sigma_{1}^{2}+b^{2}\right) Q^{2}\right]\left[\frac{\left(C_{1}+C \theta\right)(1-(Q-S))}{2 R}+\frac{\left(C_{2}+C \theta\right)(1-S)}{2 R}\right]
$$

So, estimated total cost of the system during the random cycle time with equivalent holding shortages cost is -

$\mathrm{E}\left[\mathrm{K}_{1} \mathrm{CY} / \mathrm{Q}\right]+\mathrm{EC}_{1} \mathrm{C}_{2}=$

$$
\begin{aligned}
& \frac{\left[C_{1}+C \theta+C I c-(Q-S)+. C_{2}(1-S)\right]}{2 R}\left[\sigma_{0}^{2}+\left(\sigma_{1}^{2}+b^{2}\right) Q^{2}\right]-\theta\left(C I c T^{*}\right) b Q+C[I c-I e] R T^{2}+\frac{D}{Q} C_{3} \\
& +\left[\sigma_{0}^{2}+\left(\sigma_{1}^{2}+b^{2}\right) Q^{2}\right]\left[\frac{\left(C_{1}+C \theta\right)(1-(Q-S))}{2 R}+\frac{\left(C_{2}+C \theta\right)(1-S)}{2 R}\right]
\end{aligned}
$$

Now, term (Q-S) and (1-S) multiply with (-1/2) and (1/2) corresponding and it remains (Q-S)

$$
=(Q-S)\left(\sigma_{0}^{2}+\left(\sigma_{1}^{2}+b^{2}\right) Q^{2}\right) \frac{\left[C_{1}+C \theta+C I c+. C_{2}\right]}{2 R}-\theta\left(C I c T^{*}\right) b Q+C[I c-I e] R T^{*^{2}}+C_{3} D
$$

Also, estimated duration of a cycle is ...

$\mathrm{E}(\mathrm{T}(\mathrm{y})]=\mathrm{E}[\mathrm{Y} / \mathrm{R}]=\frac{b Q}{R}$

Following silver [1976] the average estimated cost of the system per time unit is

$$
\begin{aligned}
& =T E C(Q)=\frac{E[K(Y / Q)]}{E[T(Y)]} \\
& =(Q-S) \frac{\left[C_{1}+C \theta+C I c+C_{2}\right] \sigma_{0}^{2}}{2 b Q}+\frac{\left[C_{1}+C \theta+C I c+C_{2}\right]\left(\sigma_{1}^{2}+b^{2}\right) Q}{2 b}-\theta\left(C I c T^{*} R\right)+\frac{C R^{2} T^{* 2}}{2 b Q}[I c-I e]+\frac{C_{3} D R}{b Q} \\
& =(Q-S) \frac{1}{2 b Q}\left[4 C_{3} D R+\left\{C_{1}+C \theta+C I c+. C_{2}\right\} \sigma_{0}^{2}+C[I c-I e] R^{2} T^{* 2}\right]-\theta(C I c T * R)+\frac{1}{2 b}
\end{aligned}
$$

$$
\left[C_{1}+C \theta+C I c+. C_{2}\right]\left(\sigma_{1}^{2}+b^{2}\right) Q-(8)
$$

For optimum value of $(\mathrm{Q}), \quad \frac{d E C P U T(Q)}{\partial Q}=0$ Gives

$$
\begin{aligned}
& Q^{*}=(Q-S)-\frac{\left[C_{1}+C \theta+C I c+. C_{2}\right] \sigma_{0}^{2}}{2 b Q}-\frac{C_{3} D R}{b Q^{2}}-\frac{C R^{2} T^{*}(I c-I e)}{2 b Q^{2}}+\frac{\left(C_{1}+C \theta+C I c+C_{2}\right)\left(\sigma_{1}^{2}+b^{2}\right)}{2 b}=o \\
& \therefore \frac{1}{Q^{2}}\left[(Q-S)\left(C_{1}+C \theta+C I c+. C_{2}\right] \sigma_{0}^{2}+4 C_{3} D R+C R^{2} T^{* 2}[I c-I e]=\left(C_{1}+C \theta+C I c+C_{2}\right]\left(\sigma_{1}^{2}+b^{2}\right)\right.
\end{aligned}
$$




$$
\therefore Q * \sqrt{\frac{4 C_{3} D R+(Q-S)\left[C_{1}+C \theta+C I c+. C_{2}\right] \sigma_{0}^{2}+C[I c-I e] R^{2} T^{2}}{\left[C_{1}+C \theta+C I c+. C_{2}\right]\left(\sigma_{1}^{2}+b^{2}\right)}}-
$$

At that time the economic cost per unit time is

$\operatorname{TEC}\left(Q^{*}\right)=\frac{1}{b}\left[(\mathrm{Q}-\mathrm{S})\left[\mathrm{C}_{1}+\mathrm{C} \theta+\mathrm{CIc}+\mathrm{C}_{2}\right] \sigma_{0}{ }^{2}+4 \mathrm{C}_{3} \mathrm{DR}+\mathrm{CR}^{2} \mathrm{~T}^{2} \quad[\mathrm{Ic}-\mathrm{Ie}]\left[\mathrm{C}_{1}+\mathrm{C} \theta+\mathrm{CIc}+\mathrm{C}_{2}\right]\left(\sigma_{1}{ }^{2}+\mathrm{b}^{2}\right)\right] 1 / 2-$ C Ic RT*2

To minimize the total expected cost, partial derivatives with the variable Q and S should be zero.

Taking first derivative with respect to $\mathrm{S}$ and equate it to zero.

$$
\begin{aligned}
& \frac{\partial\left(\operatorname{TEC}\left(Q^{*}\right)\right)}{\partial S}=\begin{aligned}
\frac{1}{b}\left[(\mathrm{Q}-\mathrm{S})\left[\mathrm{C}^{1}+\mathrm{C} \theta+\mathrm{CIc}+. \mathrm{C}_{2}\right] \sigma_{\circ}^{2}+4 \mathrm{C}_{3} \mathrm{DR}+\mathrm{CR}^{2} \mathrm{~T} *{ }^{2} \quad[\mathrm{Ic}-\mathrm{Ie}]\left[\mathrm{C}^{1}+\mathrm{C} \theta+\mathrm{CIc}\right.\right. \\
\left.\left.\quad+\mathrm{C}_{2}\right]\left(\sigma_{1}{ }^{2}+\mathrm{b}^{2}\right)\right] 1 / 2-\mathrm{C} \mathrm{Ic} \mathrm{RT} *^{2}
\end{aligned} \\
& =0-\left[(Q-S)\left(C_{1}+C \theta+C I c\right)+C_{2}\right] \\
& \begin{aligned}
Q\left(C_{1}+C \theta+C I c\right)=S C_{1}+S C I c+C_{2} \\
=S\left(C_{1}+C \theta+C_{2}+C I c\right)
\end{aligned} \\
& \frac{Q}{S}=\frac{C_{1}+C I c+C_{2}}{C_{1}+C I c}
\end{aligned}
$$

This is the optimum condition for $\mathrm{S}$. hence, $\mathrm{S}$ is replaced by optimum $\mathrm{S}^{*}$. now we have equation (1) and (2)

$$
\frac{Q}{S^{*}}=\frac{C_{1}+C \theta+C I c+C_{2}}{C_{1}+C \theta+C I c}-(1) \text { and } \frac{S^{*}}{Q}=\frac{C_{1}+C \theta+C I c}{C_{1}+C \theta+C I c+C_{2}}-(2)
$$

Here, RHS term is defined as settle factor for back order or shortages with interest charges

$$
F_{b}=\frac{C_{2}}{C_{1}+C \theta+C I c+C_{2}}(3)
$$

Factors are depends upon $\mathrm{C}_{1} \& \mathrm{C}_{2}$, subtracting LHs and RHs from 1.

$$
1-F_{b}=1-\frac{C_{2}}{C_{1}+C \theta+C I c+C_{2}}=\frac{C_{1}+C \theta+C I c}{C_{1}+C \theta+C I c+C_{2}}
$$

Equations (2) \& (4) $1-F_{b}=\frac{C_{1}+C \theta+C I c}{C_{1}+C \theta+C I c+C_{2}}=\frac{S^{*}}{Q}$

$\therefore \mathrm{S}^{*}=\left(1-F_{b}\right) \mathrm{Q}$

Following are some particular cases:-

If $\mathrm{T}^{*}=0$ then Ie $=\mathrm{o}$ and $\mathrm{Q}^{*}=\left[\frac{4 C_{3} D R+\left[C_{1}+C \theta+C I c+. C_{2}\right] \sigma_{o}^{2}}{\left[C_{1}+C \theta+C I c+. C_{2}\right]\left(\sigma_{1}^{2}+b^{2}\right)}\right]^{1 / 2}-$ 
$Q^{*}=\left[\frac{(Q-S)\left[C_{1}+C \theta+C I c+C_{2}\right] \sigma_{o}^{2}+4 C_{3} D R}{\left[C_{1}+C \theta+C I c+. C_{2}\right]\left(\sigma_{1}^{2}+b^{2}\right)}\right]^{1 / 2}-$

$\left.\left.\mathrm{TEC}\left(\mathrm{Q}^{*}\right)=\frac{1}{b}\left[(\mathrm{Q}-\mathrm{S})\left[\mathrm{C}_{1}+\mathrm{C} \theta+\mathrm{CIc}+. \mathrm{C}_{2}\right] \sigma_{0}^{2}+4 \mathrm{C}_{3} \mathrm{R}\right\}\left\{\mathrm{C}_{1}+\mathrm{C} \theta+\mathrm{CIc}+\mathrm{SC}_{2}\right\}\left\{\sigma_{1}^{2}+\mathrm{b}^{2}\right\}\right]\right]^{1 / 2}$

Here, if $\sigma_{1}^{2}=0$ then $\mathrm{Q}^{*}=\frac{1}{b}\left[\frac{(Q-S) 4 C_{3} D R}{\left[C_{1}+C \theta+C I c+. C_{2}\right]}+\sigma_{0}^{2}\right]^{1 / 2}-$

And,

$\operatorname{TEC}\left(Q^{*}\right)$

$$
\begin{gathered}
=\left[(\mathrm{Q}-\mathrm{S})\left[\mathrm{C}_{1}+\mathrm{C} \theta+\mathrm{CIc}+. \mathrm{C}_{2}\right] 4 \mathrm{C}_{3} \mathrm{R}+\left[\mathrm{C}_{1}+\mathrm{C} \theta+\mathrm{CIc}+\mathrm{C}_{2}\right]^{2} \sigma_{0}^{2}\right]^{1 / 2} \\
=\left[(\mathrm{Q}-\mathrm{S})\left[\mathrm{C}_{1}+\mathrm{C} \theta+\mathrm{CIc}+\mathrm{C}_{2}\right] 4 \mathrm{C}_{3} \mathrm{R}+\left[\mathrm{C}_{1}+\mathrm{C} \theta+\mathrm{CIc}+\mathrm{C}_{2}\right]^{2} \sigma_{0}^{2}\right]^{1 / 2}----
\end{gathered}
$$

And,

$\operatorname{TEC}\left(\mathrm{Q}^{*}\right)=\frac{1}{b}\left[(\mathrm{Q}-\mathrm{S})\left[\mathrm{C}_{1}+\mathrm{C} \theta+\mathrm{CIc}+\mathrm{C}_{2}\right]\left(\sigma_{1}^{2}+\mathrm{b}^{2}\right)+4 \mathrm{DC}_{3} \mathrm{R}\right] \frac{1 / 2}{1 / 2}$

If $\sigma_{\mathrm{o}}^{2}=0$ then $\mathrm{Q}^{*}=\left[\frac{(Q-S) 4 C_{3} D R}{\left[C_{1}+C \theta+C I c+. C_{2}\right]\left(\sigma_{1}^{2}+b^{2}\right)}\right]^{1 / 2}-$

Equations (14) (15) (16) (17) are as per those given by silver [1976] for a corresponding model without the permissible delay in payment.

It $\mathrm{b}=1$ and $\sigma_{\mathrm{o}}{ }^{2}=\sigma_{1}{ }^{2}=\mathrm{o}$, and $\theta=0$ then there is no uncertain in supply and in that case.

$$
Q^{*}=\left[\frac{4 C_{3} D R+C[I c-I e] R^{2} T^{* 2}}{\left[C_{1}+C I c+. C_{2}\right]}\right]^{1 / 2}-
$$

And,

$\operatorname{TEC}\left(Q^{*}\right)=\left[\left\{4 \mathrm{DC}_{3} \mathrm{R}+\mathrm{C}[\mathrm{Ic}-\mathrm{Ic}] \mathrm{R}^{2} \mathrm{~T}^{*^{2}}\left\{\left[\mathrm{C}_{1}+\mathrm{C} \theta+\mathrm{CIc}+\mathrm{C}_{2}\right]\right\}\right]^{1 / 2}-\mathrm{CIcRT}^{* 2}\right.$

Equations (18) \& (19) are as per those given by goyal [1982 ]

Here we can see that when we added Equivalent Holding \& shortages cost there is no effect on $\mathrm{C}_{3}$. Which is Replenishment cost and If there is no equivalent cost then all formulation must be same as original paper.

*Case: II - Y<RT*

In this case no any interest charges are payable the quantity reserved in stock. The total variable cost of the system in this case is given by,

Interest earned during $(0, T)$ is

$$
\begin{aligned}
& \text { CIe R } \int_{0}^{T} t d t+\left[\operatorname{CIeRT} \theta\left(T^{*}-T\right)\right] \\
& \text { CIeR }\left[\frac{T^{2}}{2}+\theta\left(T T^{*}-T^{2}\right)\right] \\
& \text { CIe }\left[T^{*} y-\theta \frac{y^{2}}{2 R}\right]
\end{aligned}
$$


$\mathrm{K}_{2}(\mathrm{Y} / \mathrm{Q})=\frac{\left[C_{1}+\left(1+\theta T^{*}\right) C I e+C \theta-(Q-S)+. C_{2}(1-S)\right] Y^{2}}{2 R}-C I e T * Y+\frac{D}{Q} C_{3}-$

Using (1) expected total cost of the system during the random cycle time is

$\mathrm{E}\left[\mathrm{K}_{2}(\mathrm{Y} / \mathrm{Q})\right]=$

$$
(Q-S) \frac{\left[C_{1}+C \theta+\left(1+\theta T^{*}\right) C I e+. C_{2}\right]}{2 R}\left(\sigma_{0}^{2}+\left(\sigma_{1}^{2}+b^{2}\right) Q^{2}\right)+\frac{D}{Q} C_{3}+C I e T^{*} b Q-
$$

we taking equivalent Holding \& shortages cost with Replenishment cost

$\mathrm{E} \mathrm{C}_{1} \mathrm{C}_{2}=\mathrm{C}_{1}(\mathrm{Q})+\mathrm{C}_{2}(\mathrm{Q})$

$$
\begin{aligned}
& =\frac{\left(C_{1}+C \theta\right)\left(Y^{2}-(Q-S)\right)}{2 R}+\frac{\left(C_{2}+C \theta\right) Y^{2}(1-S)}{2 R} \\
& =Y^{2}\left[\frac{\left(C_{1}+C \theta\right)(1-(Q-S))}{2 R}+\frac{\left(C_{2}+C \theta\right)(1-S)}{2 R}\right]
\end{aligned}
$$

Take expatiation,

$$
E\left[E C_{1} C_{2}\right]=\left[\sigma_{0}^{2}+\left(\sigma_{1}^{2}+b^{2}\right) Q^{2}\right]\left[\frac{\left(C_{1}+C \theta\right)(1-(Q-S))}{2 R}+\frac{\left(C_{2}+C \theta\right)(1-S)}{2 R}\right]
$$

So, estimated total cost of the system during the random cycle time with equivalent Holding and shortages cost is

$$
\begin{aligned}
& E\left[K_{2}(Y / Q)\right]+E C_{1} C_{2}=(Q-S) \frac{\left[C_{1}+C \theta+\left(1+\theta T^{*}\right) C I e+. C_{2}\right]}{2 R}\left[\sigma_{0}^{2}+\left(\sigma_{1}^{2}+b^{2}\right) Q^{2}\right] \\
& +C_{3}+C I e T^{*} b Q+\left[\left(\frac{\left(C_{1}+C \theta\right)(1-(Q-S))}{2 R}+\frac{\left(C_{2}+C \theta\right)(1-S)}{2 R}\right]\right. \\
& \left..\left[\sigma_{0}^{2}+\left(\sigma_{1}^{2}+b^{2}\right) Q^{2}\right]+\frac{D}{Q} C_{3}\right] \\
& =(Q-S)\left[\sigma_{0}^{2}+\left(\sigma_{1}^{2}+b^{2}\right) Q\right] \frac{\left[C_{1}+C \theta+\left(1+\theta T^{*}\right) C I e+. C_{2}\right]}{2 R}+D C_{3}+C I e T^{*} b Q-(22)
\end{aligned}
$$

The average estimated cost per time unit is

$T E C(Q)=\frac{1}{2 b Q}\left[(Q-S)\left[C_{1}+C \theta+\left(1+\theta T^{*}\right) C I e+C_{2}\right]\left[\sigma_{0}^{2}+2 C_{3} D R\right]-C I e R T^{*}+\frac{\left[C_{1}+C \theta+\left(1+\theta T^{*}\right) C I e+C_{2}\right]\left[\sigma_{1}^{2}+b^{2}\right] Q}{2 b}-\right.$

For optimum value of $\mathrm{Q} ; \frac{\partial E(P U T(Q)}{\partial Q}=0$ Gives

$$
Q^{*}=\left[\frac{2\left(Q-S^{*}\right)\left[C_{1}+C \theta+\left(1+\theta T^{*}\right) C I e+C_{2}\right]\left[\sigma_{0}^{2}+2 C_{3} D R\right.}{\left[C_{1}+C \theta+\left(1+\theta T^{*}\right) C I e+C_{2}\right]\left(\sigma_{1}^{2}+b^{2}\right)}\right]^{1 / 2}
$$

And, optimum cost per unit time is 
$\operatorname{TEC}\left(\mathrm{Q}^{*}\right)=$

$\frac{1}{b}\left[\left(Q-S^{*}\right)\left[C_{1}+C \theta+\left(1+\theta T^{*}\right) C I e+C_{2}\right]\left[\sigma_{0}^{2}+2 C_{3} D R\right]\left[C_{1}+C \theta+\left(1+\theta T^{*}\right) C I e+C_{2}\right]\left[\sigma_{1}^{2}+b^{2}\right)\right]^{1 / 2}-C I e R T^{*}-$

Some particular cases:-

If $\mathrm{T}^{*}=0$ then $\mathrm{Ie}=0$ and $\theta=0$

$\therefore Q^{*}=\left[\frac{C_{1}+C_{2}+2 C_{3} D R}{\left[C_{1}+C_{2}\right]\left(\sigma_{1}^{2}+b^{2}\right)}\right] 1 / 2$

And,

$$
\begin{gathered}
\operatorname{TEC}\left(Q^{*}\right)=\frac{1}{b}\left[\left(Q-S^{*}\right)^{2}\left\{\left[C_{1}+C \theta+C_{2}\right] \sigma_{0}^{2}+2 C_{3} D R\right]\left\{\left[C_{1}+C \theta+C_{2}\right]\left(\sigma_{1}^{2}+b^{2}\right)\right\}\right] 1 / 2 \\
=\frac{1}{b}\left[\left(Q-S^{*}\right)\left\{\left[C_{1}+C \theta+C_{2}\right] \sigma_{0}^{2}+2 C_{3} D R\right]\left(\sigma_{1}^{2}+b^{2}\right)\right] 1 /_{2}-(26)
\end{gathered}
$$

If $\sigma_{1}^{2}=0$ then,

$Q^{*}=\frac{1}{b}\left[\frac{\left(Q-S^{*}\right)+2 C_{3} D R}{\left[C_{1}+C \theta+C_{2}\right]}+\sigma_{0}^{2}\right]^{1 / 2}-$

$\operatorname{TEC}\left(\mathrm{Q}^{*}\right)=\left[\left(\mathrm{Q}-\mathrm{S}^{*}\right)\left[2\left[\mathrm{C}_{1}+\mathrm{C} \theta+\mathrm{C}_{2}+\mathrm{DC}_{3} \mathrm{R}\right]\right]+\left[\mathrm{C}_{1}{ }^{2}+\mathrm{C}_{2}{ }^{2}\right]^{\left.\sigma_{0}{ }^{2}\right] 1 / 2---(28)}\right.$

It $\quad \sigma_{0}^{2}=0$ then,

$Q^{*}=\left[\frac{2 C_{3} D R}{\left[C_{1}+C \theta+C_{2}\right]\left(\sigma_{1}^{2}+b^{2}\right)}\right]^{1 / 2}-$

$\operatorname{TEC}\left(\mathrm{Q}^{*}\right)=\frac{1}{b}\left[2\left[\mathrm{C}_{1}+\mathrm{C} \theta+\mathrm{C}_{2}+\mathrm{DC}_{3} \mathrm{R}\right]\left(\sigma_{1}^{2}+b^{2}\right)\right]^{1 / 2}-(30)$

If $\mathrm{b}=1, \sigma_{1}^{2}=1 \sigma_{0}^{2}=0$ and $\theta=0$ then in this case

$Q^{*}=\left[\frac{2 C_{3} D R}{\left[C_{1}+C_{2}+C I e\right]}\right]^{1 / 2}-(31)$

$\operatorname{TEC}\left(\mathrm{Q}^{*}\right)=\left[2\left[\mathrm{C}_{1}+\mathrm{CIe}+\mathrm{C}_{2}\right] \mathrm{DC}_{3} \mathrm{R}\right] 1 / 2-\mathrm{CIeR} \mathrm{T}^{*}$

Equations (31) \& (32) one the same as those given by Goyal [1982] and If there is no equivalent cost then all formula must be as original paper.

Now,

Total cost with interest charges due to effect of planned shortages under condition of permissible delay in payment

$\mathrm{T}(\mathrm{Q}) \quad=\mathrm{C}_{1}(\mathrm{Q})+\mathrm{C}_{2}(\mathrm{Q})+\mathrm{C}_{3}(\mathrm{Q})$ 


$$
\begin{aligned}
& =\frac{D}{Q} \cdot C_{3}+\frac{C_{1}+C \theta+C I c\left(Y^{2}-(Q-S)\right)}{2 R}+\frac{C_{2}+C \theta\left(Y^{2}-S\right)}{2 R} \\
\frac{\partial(T(Q))}{\partial S} & =\frac{\partial}{\partial S}\left[\frac{D}{Q} \cdot C_{3}+\frac{C_{1}+C \theta+C I c\left(Y^{2}-(Q-S)\right)}{2 R}+\frac{C_{2}+C \theta\left(Y^{2}-S\right)}{2 R}\right] \\
& =0-\frac{2(Y-(Q-S)) C_{1}+C \theta+C I c}{2 R}+\frac{2(Y-S) \cdot C_{2}+C \theta}{2 R}
\end{aligned}
$$

Equating it to zero

$$
\begin{aligned}
& 0=0-\frac{2(Y-(Q-S)) \cdot C_{1}+C \theta+C I c}{2 R}+\frac{2(Y-S) \cdot C_{2}+C \theta}{2 R} \\
& =-Q\left(C^{1}+C \theta+C I c\right)+S\left(C^{1}+C \theta+C I c\right)+S C_{2}+C \theta \\
& Q\left(C_{1}+C \theta+C I c\right)=S C_{1}+S\left(C_{2}+C \theta\right)+S C I c \\
& =S\left(C_{1}+C_{2}+C \theta+C I c\right) \\
& \frac{Q}{S}=\frac{C_{1}+C \theta+C I c+C_{2}}{C_{1}+C \theta+C I c}
\end{aligned}
$$

This is the optimum condition for $\mathrm{S}$. hence, $\mathrm{S}$ is replaced by optimum $\mathrm{S}^{*}$. now we have equation (1) and (2)

$$
\frac{Q}{S^{*}}=\frac{C_{1}+C \theta+C I c+C_{2}}{C_{1}+C \theta+C I c}-(1) \text { and } \frac{S^{*}}{Q}=\frac{C_{1}+C \theta+C I c}{C_{1}+C \theta+C I c+C_{2}}-(2)
$$

Here, RHS term is defined as settle factor for back order or shortages with interest charges

$$
F_{b}=\frac{C_{2}}{C_{1}+C \theta+C I c+C_{2}}(3)
$$

Factors are depends upon $\mathrm{C}_{1} \& \mathrm{C}_{2}$, subtracting LHs and RHs from 1.

$$
1-F_{b}=1-\frac{C_{2}}{C_{1}+C \theta+C I c+C_{2}}=\frac{C_{1}+C \theta+C I c}{C_{1}+C \theta+C I c+C_{2}}-
$$

Equations (2) \& (4) $1-F_{b}=\frac{C_{1}+C \theta+C I c}{C_{1}+C \theta+C I c+C_{2}}=\frac{S^{*}}{Q}$

$\therefore \mathrm{S}^{*}=\left(1-F_{b}\right) \mathrm{Q}---$

$$
F b=\frac{Q-S^{*}}{Q}=\frac{M}{Q}
$$

$\therefore \mathrm{M}=F_{b} \mathrm{Q}---(6)$

Also Equivalent Holding and shortages cost with interest charges 
$\mathrm{EC}_{1} \mathrm{C}_{2}=\mathrm{C}_{1}(\mathrm{Q})+\mathrm{C}_{2}(\mathrm{Q})$

$$
=\left[\frac{\left(C_{1}+C \theta+C I c\right)\left(Y^{2}-(Q-S)\right)}{2 R}+\frac{C_{2}+C \theta\left(Y^{2}-S\right)}{2 R}\right]
$$

Sub case put the values of $\mathrm{S}=\mathrm{S}^{*}=(1-\mathrm{Fb}) \mathrm{Q}$.

$$
\begin{aligned}
E C_{1} C_{2} & =\frac{\left(C_{1}+C \theta+C I c\right)\left(Y^{2}-(Q-S)\right)}{2 R}+\frac{C_{2}+C \theta\left(Y^{2}-S\right)}{2 R} \\
E C_{1} C_{2} & =\frac{\left(C_{1}+C \theta+C I c\right)\left(Y^{2}-(Q-S)\right)}{2 R}+\frac{C_{2}+C \theta\left(Y^{2}-S\right)}{2 R} \\
E C_{1} C_{2} & \left.\left.=\frac{1}{2 R}\left[\mathrm{Q}-\left(\mathrm{Y}^{2}-\mathrm{Fb}\right) \mathrm{Q}\right)^{2} \mathrm{C}_{1}+\mathrm{C} \theta+\mathrm{CIc}+\mathrm{C}_{2}\left(\mathrm{Y}^{2}-\mathrm{Fb}\right) \mathrm{Q}\right)^{2}\right] \\
& =\frac{1}{2 R} \mathrm{Q}^{2} \mathrm{Y}^{2}\left[\left(1-(1-\mathrm{Fb})^{2} \mathrm{C}_{1}+\mathrm{C} \theta+\mathrm{CIc}+\mathrm{C}_{2} \mathrm{Q}^{2}(1-\mathrm{Fb})^{2}\right]\right.
\end{aligned}
$$

If $\mathrm{Y}^{2}=1$ then,

$$
=\frac{Q^{2}}{2 R}\left[\mathrm{Fb}^{2} \mathrm{C}_{1}+\mathrm{C} \theta+\mathrm{CIc}+\mathrm{C}_{2}(1-\mathrm{Fb})^{2}\right]
$$

Putting the values of $\mathrm{Fb} \&(1-\mathrm{Fb})$

$$
\begin{aligned}
& =\frac{Q^{2}}{2 R}\left[\left(\frac{C_{2}}{C_{1}+C I c+C_{2}}\right)^{2} C_{1}+C \theta+C I c+C_{2}\left(\frac{C_{1}+C I c}{C_{1}+C I c+C_{2}}\right)^{2}\right] \\
& =\frac{Q^{2}}{2 R}\left[\frac{\left(C_{1}+C \theta+C I c\right) C_{2}^{2}+C_{1}^{2} \cdot C_{2}}{\left(C_{1}+C \theta+C I c+C_{2}\right)^{2}}\right] \\
& =\frac{Q^{2}}{2 R}\left[\frac{\left(C_{1}+C \theta+C I c\right) C_{2}}{C_{1}+C \theta+C I c+C_{2}}\right] \\
& =\frac{Q^{2}}{2 R} C_{1}+C \theta+C I c F b
\end{aligned}
$$

If $\mathrm{Fb}=1$ and $\theta=0 \mathrm{So}$, we can get $=\frac{Q^{2}}{2 R} C_{1}+C I c$

\section{HYPOTHETICAL NUMERICAL EXAMPLE}

\section{Numerical Illustration}

Unit cost $(\mathrm{C})=$ Rs. 20 per unit

Demand (D) = Rs.12000 unit per year
Interest charges $(\mathrm{Ic})=$ Rs.0.12 per year Replenishment cost $\left(\mathrm{C}_{3}\right)=$ Rs. 250 per order Holding cost $\left(\mathrm{C}_{1}\right)=$ Rs. 0.25 per unit per year Shortages cost $\left(\mathrm{C}_{2}\right)=$ Rs. 0.15 per unit per year 
Maximum shortages(S) = Rs. 30 per unit

Table (1) Variations in Q and TEC with changes in $\sigma_{0}^{2}$ and $\sigma_{1}^{2}$

$\mathrm{b}=0.75, \mathrm{~T}^{*}=0.085, \theta=0.01$

\begin{tabular}{|c|c|r|r|r|}
\hline $\boldsymbol{\sigma}_{1}^{\mathbf{2}} \backslash \boldsymbol{\sigma}_{0}^{\mathbf{2}}$ & & $\mathbf{5 . 0 0}$ & $\mathbf{1 0 . 0 0}$ & $\mathbf{1 5 . 0 0}$ \\
\hline \multirow{2}{*}{0.10} & $\begin{array}{c}\mathrm{Q} \\
\mathrm{TEC}(\mathrm{Q})\end{array}$ & $\begin{array}{r}353.40 \\
1928.07\end{array}$ & $\begin{array}{r}353.44 \\
1928.13\end{array}$ & $\begin{array}{r}353.45 \\
1928.19\end{array}$ \\
\hline \multirow{2}{*}{0.15} & $\mathrm{Q}$ & 340.84 & 340.85 & 340.86 \\
& $\mathrm{TEC}(\mathrm{Q})$ & 1999.58 & 1999.64 & 1999.70 \\
\hline \multirow{2}{*}{0.20} & $\mathrm{Q}$ & 329.50 & 329.51 & 329.52 \\
& TEC $(\mathrm{Q})$ & 2068.64 & 2068.70 & 2068.77 \\
\hline
\end{tabular}

Table 2. Variations in Q and TEC with changes in $\sigma_{0}^{2}$ and $\sigma_{1}^{2}$

$$
\mathrm{b}=0.75, \mathrm{~T}^{*}=0.085, \theta=0.02
$$

\begin{tabular}{|c|c|r|r|r|}
\hline $\boldsymbol{\sigma}_{1}^{2} \backslash \boldsymbol{\sigma}_{0}^{2}$ & & $\mathbf{5 . 0 0}$ & $\mathbf{1 0 . 0 0}$ & $\mathbf{1 5 . 0 0}$ \\
\hline \multirow{2}{*}{0.10} & $\mathrm{Q}$ & 348.49 & 348.40 & 348.52 \\
& $\mathrm{TEC}(\mathrm{Q})$ & 1955.37 & 1955.43 & 1955.50 \\
\hline \multirow{2}{*}{0.15} & $\mathrm{Q}$ & 336.11 & 336.12 & 336.14 \\
& $\mathrm{TEC}(\mathrm{Q})$ & 2027.97 & 2028.03 & 2028.10 \\
\hline \multirow{2}{*}{0.20} & $\mathrm{Q}$ & 324.96 & 324.97 & 324.98 \\
& TEC(Q) & 2098.11 & 2098.17 & 2098.29 \\
\hline
\end{tabular}

Table 3. Variations in Q and TEC with changes in $\sigma_{0}^{2}$ and $\sigma_{1}^{2}$

$\mathrm{b}=0.75, \mathrm{~T}^{*}=0.085, \theta=0.03$

\begin{tabular}{|c|c|r|r|r|}
\hline $\boldsymbol{\sigma}_{1}^{2} \backslash \boldsymbol{\sigma}_{0}^{2}$ & & $\mathbf{5 . 0 0}$ & $\mathbf{1 0 . 0 0}$ & $\mathbf{1 5 . 0 0}$ \\
\hline \multirow{2}{*}{0.10} & $\mathrm{Q}$ & 343.74 & 343.75 & 343.76 \\
& $\mathrm{TEC}(\mathrm{Q})$ & 1982.30 & 1982.36 & 1982.43 \\
\hline \multirow{2}{*}{0.15} & $\mathrm{Q}$ & 331.56 & 331.57 & 331.58 \\
& $\mathrm{TEC}(\mathrm{Q})$ & 055.98 & 2056.05 & 2056.11 \\
\hline \multirow{2}{*}{0.20} & $\mathrm{Q}$ & 320.60 & 320.59 & 320.58 \\
& TEC(Q) & 127.32 & 2127.25 & 2127.18 \\
\hline
\end{tabular}

Table 4. Variations in $\mathrm{Q}$ and TEC with changes in $\sigma_{0}^{2}$ and $\mathrm{T}^{*}$

$\mathrm{b}=0.75, \sigma_{1}^{2}=0.20, \theta=0.01$

\begin{tabular}{|c|c|c|r|r|}
\hline $\mathbf{T}^{*} \backslash \boldsymbol{\sigma}_{0}^{2}$ & & $\mathbf{5 . 0 0}$ & $\mathbf{1 0 . 0 0}$ & $\mathbf{1 5 . 0 0}$ \\
\hline \multirow{2}{*}{0.0417} & $\mathrm{Q}$ & 26.83 & 326.84 & 326.85 \\
& $\mathrm{TEC}(\mathrm{Q})$ & 53.51 & 2053.58 & 2053.64 \\
\hline \multirow{2}{*}{0.0833} & $\mathrm{Q}$ & 29.53 & 329.54 & 329.55 \\
& TEC(Q) & 68.79 & 2068.85 & 2068.92 \\
\hline \multirow{2}{*}{0.1250} & $\mathrm{Q}$ & 33.99 & 334.00 & 334.01 \\
& TEC(Q) & 95.10 & 2095.16 & 2095.22 \\
\hline
\end{tabular}

Table 5.Variations in Q and TEC with changes in $\sigma_{0}^{2}$ and $\mathrm{T}^{*}$

$$
\mathrm{b}=0.75, \sigma_{1}^{2}=0.20, \theta=0.02
$$

\begin{tabular}{|c|c|r|r|r|}
\hline $\mathbf{T}^{*} \boldsymbol{I}_{0}^{2}$ & & $\mathbf{5 . 0 0}$ & $\mathbf{1 0 . 0 0}$ & $\mathbf{1 5 . 0 0}$ \\
\hline \multirow{2}{*}{0.0417} & $\mathrm{Q}$ & 322.31 & 322.32 & 322.33 \\
& $\mathrm{TEC}(\mathrm{Q})$ & 2084.37 & 2084.44 & 2084.51 \\
\hline \multirow{2}{*}{0.0833} & $\mathrm{Q}$ & 324.98 & 324.99 & 325.01 \\
& $\mathrm{TEC}(\mathrm{Q})$ & 2098.25 & 2098.31 & 2098.38 \\
\hline \multirow{2}{*}{0.1250} & $\mathrm{Q}$ & 329.39 & 329.40 & 329.41 \\
& TEC(Q) & 2123.33 & 2123.39 & 2123.45 \\
\hline
\end{tabular}

Table 6. Variations in Q and TEC with changes inT*

$$
\mathrm{b}=0.75, \sigma_{1}^{2}=0.20, \theta=0.03
$$

\begin{tabular}{|c|c|c|c|c|}
\hline $\mathbf{T}^{*} \backslash \boldsymbol{\sigma}_{0}^{2}$ & & $\mathbf{5 . 0 0}$ & $\mathbf{1 0 . 0 0}$ & $\mathbf{1 5 . 0 0}$ \\
\hline \multirow{2}{*}{0.0417} & $\mathrm{Q}$ & 17.96 & 317.97 & 317.98 \\
& TEC (Q) & 14.84 & 114.91 & 114.98 \\
\hline \multirow{2}{*}{0.0833} & $\mathrm{Q}$ & 20.60 & 320.61 & 320.62 \\
& TEC (Q) & 27.31 & 127.38 & 127.45 \\
\hline \multirow{2}{*}{0.1250} & $\mathrm{Q}$ & 24.96 & 324.97 & 324.98 \\
& TEC (Q) & 51.15 & 151.22 & 151.29 \\
\hline
\end{tabular}


Table 7. Variations in $\mathrm{Q}$ and TEC with changes in $\sigma_{0}^{2}$ and $b \sigma_{0}^{2}$

$0.083, \theta=0.01$

$$
\sigma_{1}^{2}=0.20, \mathrm{M}=
$$

\begin{tabular}{|c|c|r|r|r|}
\hline $\boldsymbol{\sigma}_{0}^{2} \backslash \mathbf{b}$ & & $\mathbf{0 . 7 5}$ & $\mathbf{0 . 8 0}$ & $\mathbf{0 . 8 5}$ \\
\hline \multirow{2}{*}{5.00} & $\mathrm{Q}$ & 329.53 & 313.95 & 299.57 \\
& $\mathrm{TEC}(\mathrm{Q})$ & 2068.79 & 2035.62 & 007.73 \\
\hline \multirow{2}{*}{10.00} & $\mathrm{Q}$ & 329.54 & 313.96 & 299.58 \\
& $\mathrm{TEC}(\mathrm{Q})$ & 2068.85 & 2035.69 & 2007.79 \\
\hline \multirow{2}{*}{15.00} & $\mathrm{Q}$ & 329.55 & 313.97 & 299.59 \\
& TEC $(\mathrm{Q})$ & 2068.91 & 2035.75 & 2007.85 \\
\hline
\end{tabular}

Table 8. Variations in Q and TEC with changes in $\sigma_{0}^{2}$ and $\mathrm{b}$

$0.085, \theta=0.02$

$$
\sigma_{1}^{2}=0.20, \mathrm{~T}^{*}=
$$

Table 10. Variations in $Q$ and TEC with changes in $\sigma_{0}^{2}$ and $b$

$$
\sigma_{1}^{2}=0.20, \mathrm{~T}^{*}=0.0415, \theta=0.01
$$

\begin{tabular}{|c|c|c|c|c|}
\hline$\sigma_{0}^{2} \backslash \mathbf{b}$ & & $\mathbf{0 . 7 5}$ & $\mathbf{0 . 8 0}$ & $\mathbf{0 . 8 5}$ \\
\hline \multirow{2}{*}{5.00} & $\mathrm{Q}$ & 326.83 & 311.37 & 297.11 \\
& $\mathrm{TEC}(\mathrm{Q})$ & 2053.51 & 020.62 & 1992.95 \\
\hline \multirow{2}{*}{10.00} & $\mathrm{Q}$ & 326.84 & 311.38 & 297.12 \\
& $\mathrm{TEC}(\mathrm{Q})$ & 2053.58 & 2020.68 & 1993.01 \\
\hline \multirow{2}{*}{15.00} & $\mathrm{Q}$ & 326.85 & 311.39 & 297.13 \\
& TEC(Q) & 2053.67 & 020.75 & 1993.07 \\
\hline
\end{tabular}

Table 11. Variations in $Q$ and TEC with changes in

\begin{tabular}{|c|c|c|c|c|c|c|c|c|c|}
\hline \multirow{2}{*}{$\sigma_{0}^{2} \backslash b$} & & \multirow{2}{*}{0.75} & \multirow{2}{*}{0.80} & \multirow{2}{*}{0.85} & \multirow[b]{2}{*}{$\sigma_{0}^{2} \backslash b$} & & \multirow[b]{2}{*}{0.75} & \multirow[b]{2}{*}{0.80} & \multirow[b]{2}{*}{0.85} \\
\hline & & & & & & & & & \\
\hline \multirow{2}{*}{5.00} & $\mathrm{Q}$ & 324.98 & 309.61 & 295.42 & & & & & \\
\hline & TEC(Q) & 2098.25 & 2064.56 & 2036.23 & \multirow{2}{*}{5.00} & Q & 329.53 & 313.95 & 299.57 \\
\hline \multirow[b]{2}{*}{10.00} & $\Omega$ & 32409 & 30961 & 205.42 & & TEC(Q) & 2068.79 & 2035.62 & 2007.73 \\
\hline & TEC(Q) & 2098.31 & 2064.56 & 2036.30 & \multirow{2}{*}{10.00} & $\mathrm{Q}$ & 329.54 & 313.96 & 299.57 \\
\hline \multirow{3}{*}{15.00} & $\Omega$ & 32500 & 30063 & 20544 & & TEC(Q) & 2068.85 & 2035.69 & 2007.79 \\
\hline & TEC(Q) & 2098.38 & 2064.69 & 2036.36 & \multirow{2}{*}{15.00} & Q & 329.55 & 313.98 & 299.58 \\
\hline & & & & & & $\mathrm{TEC}(\mathrm{Q})$ & 2068.64 & 2035.75 & 2007.85 \\
\hline
\end{tabular}
$\sigma_{0}^{2}$ and $b$

$$
\sigma_{1}^{2}=0.20, \mathrm{~T}^{*}=0.085, \theta=0.01
$$

Table 9. Variations in Q and TEC with changes in $\sigma_{0}^{2}$ and $b$

$$
\sigma_{1}^{2}=0.20, \mathrm{~T}^{*}=0.085, \theta=0.03
$$

\begin{tabular}{|c|c|c|c|c|}
\hline $\boldsymbol{\sigma}_{0}^{\mathbf{2}} \backslash \mathbf{b}$ & & $\mathbf{0 . 7 5}$ & $\mathbf{0 . 8 0}$ & $\mathbf{0 . 8 5}$ \\
\hline \multirow{2}{*}{5.00} & $\mathrm{Q}$ & 320.60 & 305.42 & 291.42 \\
& $\mathrm{TEC}(\mathrm{Q})$ & 2127.31 & 2093.11 & 2064.35 \\
\hline \multirow{2}{*}{10.00} & $\mathrm{Q}$ & 320.61 & 305.43 & 291.43 \\
& $\mathrm{TEC}(\mathrm{Q})$ & 2127.38 & 2093.18 & 2064.42 \\
\hline \multirow{2}{*}{15.00} & $\mathrm{Q}$ & 320.62 & 305.44 & 291.44 \\
& TEC(Q) & 2127.45 & 2093.25 & 2064.49 \\
\hline
\end{tabular}

Table 12. Variations in $Q$ and TEC with changes in $\sigma_{0}^{2}$ and $b$

$$
\sigma_{1}^{2}=0.20, \mathrm{~T}^{*}=0.127, \theta=0.01
$$

\begin{tabular}{|c|c|c|c|c|}
\hline $\boldsymbol{\sigma}_{0}^{\mathbf{2}} \backslash \boldsymbol{b}$ & & $\mathbf{0 . 7 5}$ & $\mathbf{0 . 8 0}$ & $\mathbf{0 . 8 5}$ \\
\hline 5.00 & $\begin{array}{c}\mathrm{Q} \\
\text { TEC(Q) }\end{array}$ & 333.99 & 318.20 & 303.62 \\
& $\mathrm{Q}$ & 334.09 & 2061.48 & 2033.21 \\
\hline 10.00 & $\mathrm{TEC}(\mathrm{Q})$ & 095.16 & 2061.54 & 2033.27 \\
\hline 15.00 & $\mathrm{Q}$ & 334.01 & 318.21 & 303.64 \\
& TEC(Q) & 095.22 & 2061.61 & 2033.33 \\
\hline
\end{tabular}


Table 13. Variations in $\mathrm{Q}$ and TEC with changes in $\sigma_{1}^{2}$ and $b$

$$
\sigma_{0}^{2}=5.00, \mathrm{~T}^{*}=0.085, \theta=0.01
$$

\begin{tabular}{|c|c|r|c|c|}
\hline $\boldsymbol{\sigma}_{1}^{2} \backslash \mathbf{b}$ & & $\mathbf{0 . 7 5}$ & $\mathbf{0 . 8 0}$ & $\mathbf{0 . 8 5}$ \\
\hline \multirow{2}{*}{0.10} & $\mathrm{Q}$ & 353.46 & 334.43 & 317.20 \\
& $\mathrm{TEC}(\mathrm{Q})$ & 1928.21 & 910.49 & 1895.68 \\
\hline \multirow{2}{*}{0.15} & $\mathrm{Q}$ & 340.87 & 323.70 & 308.01 \\
& $\mathrm{TEC}(\mathrm{Q})$ & 1999.72 & 974.04 & 1952.50 \\
\hline \multirow{2}{*}{0.20} & $\mathrm{Q}$ & 329.53 & 313.95 & 299.57 \\
& $\mathrm{TEC}(\mathrm{Q})$ & 2068.79 & 2035.62 & 2007.73 \\
\hline
\end{tabular}

Table 14. Variations in $\mathrm{Q}$ and TEC with changes in $\sigma_{1}^{2}$ and $b$

$$
\sigma_{0}^{2}=5.00, \mathrm{~T}^{*}=0.085, \theta=0.02
$$

\begin{tabular}{|c|c|c|c|c|}
\hline $\boldsymbol{\sigma}_{1}^{2} \backslash \mathbf{b}$ & & $\mathbf{0 . 7 5}$ & $\mathbf{0 . 8 0}$ & $\mathbf{0 . 8 5}$ \\
\hline \multirow{2}{*}{0.10} & $\mathrm{Q}$ & 348.52 & 329.75 & 312.76 \\
& TEC $(\mathrm{Q})$ & 1955.50 & 937.52 & 1922.49 \\
\hline \multirow{2}{*}{0.15} & $\mathrm{Q}$ & 336.14 & 319.21 & 303.72 \\
& TEC $(\mathrm{Q})$ & 2028.10 & 2002.03 & 1980.16 \\
\hline \multirow{2}{*}{0.20} & $\mathrm{Q}$ & 324.98 & 309.61 & 295.42 \\
& TEC $(\mathrm{Q})$ & 2098.25 & 2064.56 & 2036.23 \\
\hline
\end{tabular}

Table 15. Variations in $\mathrm{Q}$ and TEC with changes in $\sigma_{1}^{2}$ and $b$

$$
\sigma_{0}^{2}=5.00, \mathrm{~T}^{*}=0.085, \theta=0.03
$$

\begin{tabular}{|c|c|r|r|r|}
\hline $\boldsymbol{\sigma}_{1}^{2} \backslash \mathbf{b}$ & & $\mathbf{0 . 7 5}$ & $\mathbf{0 . 8 0}$ & $\mathbf{0 . 8 5}$ \\
\hline \multirow{2}{*}{0.10} & $\mathrm{Q}$ & 343.77 & 325.24 & 308.47 \\
& TEC(Q) & 1982.42 & 1964.17 & 1948.92 \\
\hline \multirow{2}{*}{0.15} & $\mathrm{Q}$ & 331.59 & 314.87 & 291.42 \\
& TEC $(\mathrm{Q})$ & 2056.10 & 2029.64 & 2007.44 \\
\hline \multirow{2}{*}{0.20} & $\mathrm{Q}$ & 320.61 & 305.43 & 291.42 \\
& TEC (Q) & 2127.31 & 2093.11 & 2064.36 \\
\hline
\end{tabular}

Table 16. Variations in $\mathrm{Q}$ and TEC with changes in $\sigma_{1}^{2}$ and $b$

$$
\sigma_{0}^{2}=5.00, \mathrm{~T}^{*}=0.0415, \theta=0.01
$$

\begin{tabular}{|c|c|r|r|r|}
\hline$\sigma_{1}^{2} \backslash \mathbf{b}$ & & $\mathbf{0 . 7 5}$ & $\mathbf{0 . 8 0}$ & $\mathbf{0 . 8 5}$ \\
& & & & \\
\hline & $\mathrm{Q}$ & 350.56 & 331.69 & 314.60 \\
0.10 & $\mathrm{TEC}(\mathrm{Q}$ & 1914.0 & 1896.5 & 1881.8 \\
& ) & 8 & 1 & 2 \\
\hline & $\mathrm{Q}$ & 338.07 & 321.05 & 305.49 \\
0.15 & $\mathrm{TEC}(\mathrm{Q}$ & 1985.0 & 1959.5 & 1938.1 \\
& ) & 1 & 4 & 8 \\
\hline & $\mathrm{Q}$ & 326.83 & 311.37 & 297.12 \\
0.20 & $\mathrm{TEC}(\mathrm{Q}$ & 2053.5 & 2020.6 & 1992.9 \\
& ) & 1 & 2 & 5 \\
\hline
\end{tabular}

\begin{tabular}{|c|c|c|c|c|}
\hline$\sigma_{1}^{2} \backslash b$ & & 0.75 & 0.80 & 0.85 \\
\hline \multirow{2}{*}{0.10} & $\mathrm{Q}$ & 353.46 & 334.43 & 317.20 \\
\hline & TEC(Q) & 928.21 & 910.49 & 1895.68 \\
\hline \multirow{2}{*}{0.15} & $\mathrm{Q}$ & 340.85 & 323.70 & 308.01 \\
\hline & $\operatorname{TEC}(\mathrm{Q})$ & 999.72 & 974.04 & 1952.50 \\
\hline \multirow{2}{*}{0.20} & $\mathrm{Q}$ & 329.53 & 313.95 & 299.57 \\
\hline & $\mathrm{TEC}(\mathrm{Q})$ & 068.79 & 035.62 & 2007.73 \\
\hline
\end{tabular}

Table 17. Variations in $\mathrm{Q}$ and TEC with changes in $\sigma_{1}^{2}$ and $b$

$$
\sigma_{0}^{2}=5.00, \mathrm{~T}^{*}=0.085, \theta=0.02
$$

Table 18. Variations in $\mathrm{Q}$ and TEC with changes in $\sigma_{1}^{2}$ and $b$

$$
\sigma_{0}^{2}=5.00, \mathrm{~T}^{*}=0.085, \theta=0.03
$$

\begin{tabular}{|c|c|c|c|c|}
\hline $\boldsymbol{\sigma}_{1}^{2} \backslash \mathbf{b}$ & & $\mathbf{0 . 7 5}$ & $\mathbf{0 . 8 0}$ & $\mathbf{0 . 8 5}$ \\
& & & & \\
\hline \multirow{2}{*}{0.10} & $\mathrm{Q}$ & 358.24 & 338.95 & 321.49 \\
& $\mathrm{TEC}(\mathrm{Q})$ & 1952.62 & 1934.66 & 1919.66 \\
\hline \multirow{2}{*}{0.15} & $\mathrm{Q}$ & 345.48 & 328.08 & 312.18 \\
& $\mathrm{TEC}(\mathrm{Q})$ & 2025.10 & 1999.07 & 1977.24 \\
\hline \multirow{2}{*}{0.20} & $\mathrm{Q}$ & 333.99 & 318.20 & 303.62 \\
& TEC $(\mathrm{Q})$ & 2095.10 & 2061.48 & 2033.21 \\
\hline
\end{tabular}




\section{Interpretations}

In Tables $1-3$, we study the effects of $\sigma_{0}^{2}$ and $\sigma_{1}^{2}$ keeping $\mathrm{b}$ constant and varying deterioration rate $\theta$ of the units in inventory. It is found that as $\sigma_{0}^{2}$ increases, the optimum purchase quantity and total expected total cost of the system increases whereas increase in $\sigma_{1}^{2}$ results decrease in optimum procurement quantity and increase average expected total cost of the system. With increase in deterioration of units, optimum purchase quantity decreases and cost of an inventory system increases.

- In Tables $4-6$, the effects of variations in $\sigma_{0}^{2}$ and delay period $\mathrm{T}^{*}$ have been studied keeping $\sigma_{1}^{2}$ and $\mathrm{b}$ constant and varying deterioration rate of the units in inventory. Increase in $\sigma_{0}^{2}$ results increase in both, optimum purchase quantity and total expected cost. Same pattern is observed when delay in payment period increases.

- In Tables $7-8$, the effects of $\mathrm{b}$ and $\sigma_{0}{ }^{2}$ when $\sigma_{1}{ }^{2}$ and delay period are fixed and deterioration rate of units increases. It is found that increase in $b$ results decrease in optimum procurement quantity and total expected cost of an inventory system. As $\sigma_{0}{ }^{2}$ increases, optimum purchase quantity and total expected cost increases. While as deterioration rate increases, number of units to be procured decrease and total cost of the system increases. When delay period increases, there is increase in procurement units and expected total cost.

- The effects of variations in deterioration, $b$ and $\sigma_{1}{ }^{2}$ have been studied on optimum purchase quantity and total expected cost in tables 13 - 15. It is observed that increase in $b$ results decrease in optimum purchase units and total expected cost, whereas increase in $\sigma_{1}^{2}$ results decrease in purchase quantity and increase in total expected cost. Also, when deterioration of units in inventory increase optimum procurement quantity decreases and total expected cost increases.

- In Tables 16 - 18, we study variations of delay period, bias factor $\mathrm{b}$ and $\sigma_{1}^{2}$ have been studied on optimum purchase quantity and total expected cost. It is observed that increase in b results decrease in optimum purchase units and total expected cost, whereas increase in $\sigma_{1}^{2}$ results decrease in purchase quantity and increase in total expected cost. When delay period increases, optimum purchase quantity and total expected cost of an inventory system increases.

\section{References}

[1] COVERT, R.P. and G.C. PHILIP (1973): “An EOQ model for items with weibull distribution deterioration", AIIE Trans., 6, 323-326.

[2] GHARE, P.M. and G.F. SCHRADER (1963): "A model for exponentially decaying inventory", Jr. of Indus. Engg., 14, 238-243.

[3] GOYAL, S.K. (1985): "EOQ under conditions of permissible delay in payment", JORS, 36(4),

335-338.

[4] KALRO, A.H. and M.M. GOHIL (1982): “A lot-size model with backlogging when amounts received is uncertain", IJPR 20(6), 775-786.

[5] MANDAL, B.N. and S. PHAUJDAR (1989): "Some EOQ models under permissible delay in payments", IJOMAS 5(2), 99108.

[6] NOORI, A.H. and KELLER (1986): "The lot-size reorder model with upstreamdownstream uncertainty", Deci. Sci. 17, 285-291.

[7] PHILIP, G.C. (1974) : A generalized EOQ model for items with weibull distribution deterioration, AIIE Trans., 6, 159-162.

[8] SILVER, E.A. (1976): "Establishing the order quantity when the amount received is uncertain", INFOR 14(1), 32 39. 